\title{
Calculation of the quark-quark contribution to the Drell-Yan process
}

\author{
A. N. Schellekens \\ Institute for Theoretical Physics, Nijmegen, The Netherlands \\ W. L. van Neerven \\ CERN, Geneva, Switzerland \\ (Received 7 June 1979; revised manuscript received 21 September 1979)
}

\begin{abstract}
We calculate the quark-quark contribution to lepton-pair production, which was argued to give a large contribution in proton-proton scattering. Also the corresponding deep-inelastic scattering diagrams are calculated. Factorization and universality are explicitly demonstrated; and the results are shown to be independent of the way the mass singularities are regularized. We find that the correct scale for the distribution functions used in the Drell-Yan process is $Q^{2}$. The quark-quark contribution is found to be dominant for large values of $\tau$.
\end{abstract}

\section{INTRODUCTION}

Recently, a number of authors ${ }^{1}$ have shown that the mass singularities appearing in perturbative quantum chromodynamics are factorizable and can be absorbed in the parton distribution functions. In the case of lepton-pair production in protonproton scattering, this implies that the parton distribution functions in the well-known Drell-Yan formula ${ }^{2}$ are modified to scale-dependent distribution functions, which are obtained by summing up the mass singularities using renormalizationgroup methods. These distribution functions are. equal to the ones measured in deep-inelastic scattering processes.

However, this scaling violation is not the only effect of higher-order corrections. After the removal of the mass singularities, finite terms remain, which modify the leading quark-antiquark annihilation process by powers in the running coupling constant $\alpha_{s}$, or can be interpreted as contributions to other parton-parton subprocesses.

Although these finite correction terms are suppressed by powers of $\alpha_{s}$ compared to the leading process, it has been argued ${ }^{3}$ that in proton-proton scattering a number of parton-parton subprocesses are equally important for lepton-pair production. Beside the zeroth-order process $q \bar{q} \rightarrow \gamma$, these are the first-order process $q g \rightarrow q \gamma$ and the second-order process $q q \rightarrow q q \gamma$. These processes are expected to be important due to the fact that in the high $-\tau=Q^{2} / s$ region (which implies high $x$ ), the valence-quark distribution function is much larger than the gluon distribution function which, in turn, is larger than the sea-quark distribution functions.

Because $\alpha_{s}\left(Q^{2}\right)$ is not very small over a large range of $Q^{2}$ one should, however, be careful with such an order-of-magnitude estimate. It may happen that processes not mentioned here, because they are expected to give only a small contribution, turn out to be important as well. This is found ${ }^{4}$ to be the case for the process $q \bar{q} \rightarrow \gamma g$, which overwhelms the leading-order process due to an unexpectedly large contribution of final and virtual gluons.

This paper is concerned with a calculation of the third process mentioned above, the $q q \rightarrow q q \gamma$ contribution. The second process $q g \rightarrow q \gamma$ has already been calculated. ${ }^{5}$ We obtain this quark-gluon cross section as an intermediate result.

Besides the calculation of the cross section, our purpose is first of all to show, in a nontrivial case, that factorization and universality hold without unforeseen difficulties. Since we have to deal with factorization of nonleading logarithms, we are able to determine the correct variable in terms of which scaling deviations should be formulated, if deep-inelastic scattering distributions are used for the Drell-Yan process. As was remarked by Kubar-André and Paige ${ }^{5}$ cancellation of the leading logarithms alone does not determine the scale of this variable. This scale might even have been moment-dependent. We will, however, show that the correct variable is $Q^{2}$.

Further, we have explicitly checked that the final results which are obtained are independent of the way the mass singularities are regularized. To show this we have calculated all our results both with massless, off-shell initial quarks and with massive, on-shell quarks.

We will restrict ourselves to nonidentical quarks. If the quarks are identical there is also a contribution due to "crossed" diagrams, but they will not alter the conclusions concerning the points mentioned above. We also have no reason to expect that they will significantly change the magnitude of the $q q$ contribution, but of course they have to be calculated when the lepton-pair production data be- 
come sufficiently accurate. If necessary, our calculation methods can be extended to the identicalquark contribution.

The organization of this paper is as follows. In the following section we discuss how factorization is expected to work for this process and how an unambiguous result can be obtained. In Sec. III we present the calculation and the results of the quark-quark diagrams. 'In Sec. IV the deep-inelastic scattering diagrams needed for the subtraction of the singularities are calculated, and in Sec. $V$ the regularization dependence of the quarkgluon transition function is investigated. Then the results of Secs. III-V are combined in Sec. VI. Finally, we give some numerical results.

\section{FACTORIZATION}

The starting point for perturbative calculations in lepton-pair production is the relation between the hadron cross section and the parton cross sections:

$$
\begin{array}{rl}
\frac{d \sigma}{d Q^{2}}=\sum_{i, j} \int_{0}^{1} & d x_{1} d x_{2} \theta\left(x_{1} x_{2}-\tau\right) \\
& \times f_{i}\left(x_{1}\right) f_{j}\left(x_{2}\right) \frac{d \sigma^{i j}}{d Q^{2}}\left(\frac{\tau}{x_{1} x_{2}}\right) .
\end{array}
$$

Here, $x_{k}$ is the momentum fraction carried by a parton in hadron $k, f_{i}\left(x_{k}\right)$ is the momentum distribution function for parton $i$ in hadron $k$, and $d \sigma^{i j} / d Q^{2}$ is the perturbatively calculated cross section for the process $i+j \rightarrow \gamma+$ anything. The distribution functions appearing in (2.1) are the "bare" ones, defined at a fixed subtraction point $p_{k}^{2}$.

When the parton cross sections are calculated, powers of $\ln \left(p_{k}{ }^{2} / Q^{2}\right)$ appear which spoil perturbation theory in the asymptotic freedom limit. However, it is possible to factorize these logarithms out of the parton cross sections. The most elegant way to do this is by taking moments. ${ }^{3}$ Using the formulation of Politzer ${ }^{1}$ we define these moments as follows ${ }^{6}$ :

$$
\begin{aligned}
& \sigma_{i j}^{n}=\int_{0}^{1} d \tau \tau^{n} \sigma_{i j}(\tau), \\
& f_{i}^{n}=\int_{0}^{1} d x x^{n+1} f_{i}(x) .
\end{aligned}
$$

Inserting these expressions in (2.1) one finds

$$
\sigma^{n}=\sum_{i, j} f_{i}^{n} f_{j}^{n} \sigma_{i j}^{n}
$$

Factorization can now be stated as follows:

$$
\begin{aligned}
\sigma_{i j}^{n} & \left(\ln \frac{p_{1}^{2}}{Q^{2}}, \ln \frac{p_{2}{ }^{2}}{Q^{2}}\right) \\
& =\sum_{i^{\prime}, j^{\prime}} \sigma_{i^{\prime} j^{\prime}}^{n}(0,0) \Gamma_{i^{\prime} i}^{n}\left(\ln \frac{p_{1}{ }^{2}}{Q^{2}}\right) \Gamma_{j^{\prime}, j}^{n}\left(\ln \frac{p_{2}^{2}}{Q^{2}}\right) .
\end{aligned}
$$

Now (2.3) can be substituted in (2.2) to obtain

$$
\sigma^{n}=\sum_{i, j} \sum_{i^{\prime}, j^{j}}\left[\left(\Gamma_{i^{\prime} i}^{n}\left(\ln \frac{p_{1}^{2}}{Q^{2}}\right) f_{i}^{n}\right]\left[\Gamma_{j j^{\prime} j}^{n}\left(\ln \frac{p_{2}{ }^{2}}{Q^{2}}\right) f_{j}^{n}\right] \sigma_{i^{\prime} j^{\prime}}^{n} .\right.
$$

The terms between brackets are the moments of the $Q^{2}$-dependent distribution functions.

To formulate factorization for the process $q_{1} q_{2} \rightarrow q_{1} q_{2} \gamma$ we split the cross section in three parts, proportional to $e_{1}{ }^{2}, e_{2}{ }^{2}$, and $e_{1} e_{2}$, respectively. Here $e_{i}$ is the charge of parton $i$. The cross section can be written as follows:

$$
\begin{aligned}
\sigma_{q 1 Q 2}^{n}( & \left.\ln \frac{p_{1}{ }^{2}}{Q^{2}}, \ln \frac{p_{2}{ }_{2}}{Q^{2}}\right) \\
= & e_{1}^{2} \sigma_{1}^{n}\left(\ln \frac{p_{2}{ }^{2}}{Q^{2}}\right)+e_{2}^{2} \sigma_{2}^{n}\left(\ln \frac{p_{1}{ }^{2}}{Q^{2}}\right)+2 e_{1} e_{2} \sigma_{1}^{n} .
\end{aligned}
$$

The functions $\sigma_{1}^{n}$ and $\sigma_{2}^{n}$ are, of course, identical. Now the logarithms factorize in the following way:

$$
\begin{aligned}
\sigma_{q 1 q 2}^{n}\left(\ln \frac{p_{1}{ }^{2}}{Q^{2}}\right. & \left., \ln \frac{p_{2}{ }^{2}}{Q^{2}}\right) \\
= & e_{1}{ }^{2} \sigma_{q 1 \bar{q} 1}^{n} \Gamma_{\bar{q} 1 q 2}^{n}\left(\ln \frac{p_{2}{ }^{2}}{Q^{2}}\right)+e_{2}{ }^{2} \sigma_{q_{2 q_{2}}}^{n} \Gamma_{q_{2} q_{1}}^{n}\left(\ln \frac{p_{1}{ }^{2}}{Q^{2}}\right) \\
& +e_{1}{ }^{2} \sigma_{q 1 g}^{n} \Gamma_{g \alpha_{2}}^{n}\left(\ln \frac{p_{2}{ }^{2}}{Q^{2}}\right)+e_{2}{ }^{2} \sigma_{q_{2} g}^{n} \Gamma_{g q_{1}}^{n}\left(\ln \frac{p_{1}{ }^{2}}{Q^{2}}\right) \\
& +e_{1}{ }^{2} \sigma_{1}^{n}(0,0)+e_{2}{ }^{2} \sigma_{2}^{n}(0,0)+2 e_{1} e_{2} \sigma_{I}^{n} .
\end{aligned}
$$

The cross sections on the right-hand side of (2.6) are the singularity-free contributions of the lowerorder processes. In this way, the unwanted logarithms in $q_{1} q_{2}-q_{1} q_{2} \gamma$ contribute to the scaling violating terms in the antiquark and gluon distribution functions of $q \bar{q} \rightarrow \gamma$ and $q g \rightarrow q \gamma$.

The factorization of logarithms described here is by no means a unique procedure, since an arbitrary constant can be factored out together with the logarithms. This fact is well-known in deep-inelastic scattering, analyzed by means of the operator-product expansion. ${ }^{7}$ This freedom of definition corresponds in that case to finite renormalization of the matrix elements of the operators.

In order to compare results obtained in deepinelastic scattering and lepton-pair production, one must use the same convention in both processes and absorb the same constants in the bare distribution functions, which are process independent by assumption. The conventions chosen in both processes can only be related with each other if the corresponding diagrams for deep-inelastic scattering are also calculated. For the process under consideration these are the diagrams of Fig. 1 , involving quark-antiquark transitions, and a diagram for the quark-gluon transition (Fig. 2). 

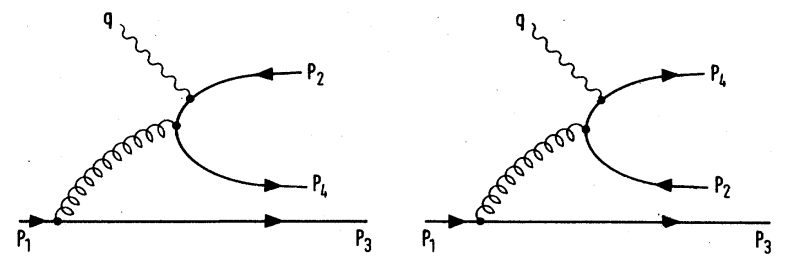

FIG. 1. Diagrams contributing to the sea-quark distribution functions.

For the subtraction of the singular terms we adopt the convention which was used in most of the calculations of the $q g$ process. ${ }^{5}$ According to this convention, the quark and antiquark distributions are defined by the requirement that the following relation, which is valid in leading order, is not affected by higher-order corrections:

$$
\nu W_{2}\left(x, Q^{2}\right)=\sum_{i} q_{i}^{2} x\left[f_{i}\left(x, Q^{2}\right)+\bar{f}_{i}\left(x, Q^{2}\right)\right],
$$

( $\nu=P \cdot q / M$, where $P^{\mu}$ and $M$ are the four-momentum and mass of the proton and $\bar{f}_{i}$ denotes the antiquark distribution function.) This implies that all corrections are to be absorbed in the distribution functions. Unfortunately, the gluon distribution does not appear in (2.7) and therefore one has to look for a different process to define and measure this distribution. At present we do not know a clear candidate for such a process, and therefore the subtraction of the quark-gluon mass singularity remains necessarily a somewhat arbitrary procedure. To estimate the magnitude of the quarkquark contribution we have to make a choice, but we are aware of the fact that it is a priori not clear that this choice matches the gluon distribution function we use.

For the antiquark distributions the situation is slightly better because they can be confronted directly with the experimental results for $\nu W_{2}$ using (2.7). For example, the Buras-Gaemers ${ }^{8}$ distributions, which we will use, show very reasonable agreement with these results.

Of course, other choices for the absorption of the singularities in the distribution functions are possible. One can, for example, define the distribution functions by means of an expression in terms of structure functions for neutrino scattering. All other definitions differ by powers of $\alpha_{s}$

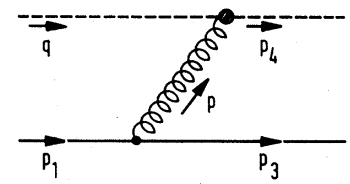

FIG. 2. Diagram used for the calculation of the quarkgluon transition function. The dashed line represents the dummy scalar particle. from our choice, and all our results have to be modified if alternatively defined distribution functions are used. To allow for difference choices we present all our results in their complete analytic form.

We will obviously encounter the convention dependence mentioned above if the mass singularities are regularized in different ways. In the literature two methods have been used. The first one is to take the ingoing quarks off mass shell. Then the squares of the ingoing momenta are used as a mass scale for the logarithms, and all internal quark masses are neglected. The second one is to take the ingoing particles on shell and to use the quark masses themselves to set the scale. We have considered both methods. As expected, they yield completely different results for the diagrams of Figs. 1 and 3 separately, but it is possible to find the relation between on-shell and off-shell distribution functions which compensates for this difference. To check this, the relevant deep-inelastic scattering diagrams must be calculated using both methods, with the same definition (2.7). Because both the distribution functions, obtained from an off-shell calculations and the on-shell distribution functions, satisfy (2.7), they must be identical. Of course, the unrenormalized distribution functions are different in these two cases. We have shown explicitly that this different is universal in the same sense as the mass singularities.

\section{CALCULATION OF THE LEPTON-PAIR PRODUCTION DIAGRAMS}

The quark-quark cross section we are calculating consists of two parts, as was shown in (2.5). These are the "squared" terms, proportional to the square of one of the charges, and the interference term, proportional to the product of the two
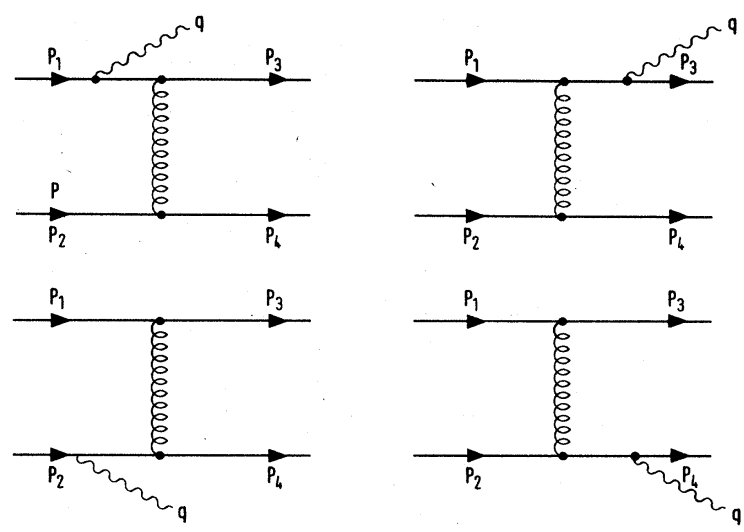

FIG. 3. The quark-quark contribution. Gluons are denoted by curly lines, photons by wavy lines. 
charges. These two parts are rather different in character. The squared terms have a double logarithmic mass singularity and consequently the nonleading logarithms and the finite constants are not unique. The interference terms must be singularity-free, since there is no way to absorb mass singularities proportional to $e_{1} e_{2}$. This implies that the contribution of the interference terms is unambiguous, because arbitrary constants can only be introduced via the factorization of singular terms. Notice that the $\Gamma$ factors cannot depend upon quark charges, because their universality should extend to processes without electromagnetic coupling.

For the squared terms the off-shell method described in the previous paragraph turned out to give the simplest result. Therefore, we first discuss the off-shell calculation of the squared terms, then the calculation of the interference terms, and finally mention some aspects of the on-shell calculation.

\section{A. Squared terms (off-shell calculation)}

We will consider the terms proportional to $e_{2}{ }^{2}$ and choose the Feynman gauge. Then the squared matrix element can be written as follows (the momenta are defined in Fig. 3):

$$
\sum|M|^{2}=A^{\mu \nu}\left(p_{1}, p_{3}\right) B_{\mu \nu}\left(p_{2}, p_{4}, q\right) \text {. }
$$

The summation symbol denotes both spin and color summation. The tensor $A^{\mu \nu}$ is defined as follows:

$$
A^{\mu \nu}=\frac{4}{\left(p^{2}\right)^{2}}\left(p_{3}^{\mu} p_{1}^{\nu}+p_{1}^{\mu} p_{3}^{\nu}-g^{\mu \nu} p_{1} \cdot p_{3}\right),
$$

where $p^{\mu}=p_{1}^{\mu}-p_{3}^{\mu}$.

To calculate the phase-space integral, we will first consider the subprocess $g\left(p^{\mu}\right)+q_{2}\left(p_{2}^{\mu}\right) \rightarrow \gamma\left(q^{\mu}\right)$ $+q_{2}\left(p_{4}^{\mu}\right)$. The tensor $B_{u \nu}$ is the sum of the four traces contributing to this process. When the phase-space integral for the subprocess is performed one obtains a tensor $T_{\mu \nu}$, which can be decomposed in four structure functions:

$$
\begin{aligned}
T_{\mu \nu}= & \int \frac{d^{3} p_{4}}{(2 \pi)^{3} 2 p_{4}^{0}} \frac{d^{3} q}{(2 \pi)^{3} 2 q^{0}} \\
& \times(2 \pi)^{4} \delta^{4}\left(p+p_{2}-p_{4}-q\right) B_{\mu \nu}\left(p_{2}, p_{4}, q\right), \\
T_{\mu \nu}= & T_{1}\left[-g_{\mu \nu}+\frac{p_{\mu} p_{\nu}}{p^{2}}\right] \\
& +\frac{T_{2}}{p_{2}^{2}}\left[p_{2 \mu}-\frac{p_{2} \cdot p^{2}}{p^{2}} p_{\mu}\right]\left[p_{2 \nu}-\frac{p_{2} \cdot p}{p^{2}} p_{\nu}\right] \\
& +T_{3} p_{\mu} p_{\nu}+T_{4}\left(p_{\mu} p_{2 \nu}+p_{2 \mu} p_{\nu}\right) .
\end{aligned}
$$

The structure functions $T_{1}, \ldots, T_{4}$ are scalar functions of the two Lorentz invariants $p^{2}$ and $s_{2}$ $=\left(p+p_{2}\right)^{2}$. For a gauge invariant tensor $T_{3}$ and $T_{4}$ would vanish, but since we are calculating an offshell cross section this is not the case. To calculate the structure functions we use the following projections:

$$
\begin{aligned}
& C=g^{\mu \nu} T_{\mu \nu}, \\
& D=p_{2}^{\mu} p_{2}^{\nu} T_{\mu \nu}, \\
& E=p^{\mu} p^{\nu} T_{\mu \nu} . \\
& F=\left(p_{2}^{\mu} p^{\nu}+p^{\mu} p_{2}^{\nu}\right) T_{\mu \nu} .
\end{aligned}
$$

The calculation of these terms can most easily be done in the c.m. frame of $p^{\mu}$ and $p_{2}^{\mu}$. Then, only an integral over a single angle remains to be calculated. Since only one propagator depends on this angle this integral is trivial.

Now we use (3.1)-(3.4) to express the integrand for the total process in $C, \ldots, F$. For the phasespace integral we find

$$
\begin{aligned}
\int d \odot \sum|M|^{2}=\frac{1}{4 s} \frac{1}{(2 \pi)^{2}} \int_{Q^{2}}^{s} d s_{2} \int_{\left(s_{2}-s\right)}^{s_{2} p_{1}{ }^{2} / s} d p^{2}\{ & {\left[1+\frac{\left(p_{2} \cdot k\right)^{2}}{\beta}-2 \frac{p_{1}{ }^{2}}{p^{2}} \frac{\left(p_{2} \cdot k\right)\left(p_{2} \cdot p\right)}{\beta}\right] \frac{C}{p^{2}} } \\
& +\left[1-\frac{3\left(p_{2} \cdot k\right)^{2}}{\beta}+6 \frac{p_{1}{ }^{2}}{p^{2}} \frac{\left(p_{2} \cdot k\right)\left(p_{2} \cdot p\right)}{\beta}+\frac{2 p_{1}{ }^{2}}{p^{2}}-\frac{2 p_{1}{ }^{4}}{\left(p^{2}\right)^{2}}\right] \frac{D}{\beta} \\
& \left.+\left[-1+\frac{\left(p_{2} \cdot k\right)}{\beta}\right] \frac{E}{\left(p^{2}\right)^{2}}\right\}
\end{aligned}
$$

Here

$$
k^{\mu}=p_{1}^{\mu}+p_{3}^{\mu} \quad \beta=\frac{1}{4}\left[\left(s_{2}+p^{2}-p_{2}^{2}\right)^{2}-4 s_{2} p^{2}\right] .
$$

Notice that the singular behavior of this integral is completely determined by the upper boundary of the $p^{2}$ integral. Near $p^{2}=0$ the factor $C$ is logarithmically divergent, $D$ diverges as $1 / p^{2}$, and $E$ is finite. Since the result is gauge-invariant for $p_{2}^{2}=0, E$ is proportional to $p_{2}{ }^{2}$.

The terms proportional to $E$ require special attention. In the final result they yield terms containing ratios of $p_{2}{ }^{2}$ and $p_{1}{ }^{2}$. Such terms are arbitrary and phenomenologically unacceptable, but they can be absorbed in the distribution functions if the same terms appear in the result of the diagrams of Fig. 1. To achieve that, the outgoing an- 
tiquark must be taken off shell like the ingoing quark with momentum $p_{2}$ in Fig. 3 .

Of course, an easier method with the same final result is to drop these terms directly by taking $p_{2}{ }^{2}=0$. This is possible because ${p_{1}}^{2}$, not ${p_{2}}^{2}$, appears as an argument of the logarithms. Since neglecting $E$ is equivalent to neglecting $T_{3}$ and $T_{4}$, we get a gauge-independent result by dropping these terms.

The integrals in (3.6) which remain to be calculated are separately straightforward, but very large in number. To evaluate the double integral over $p^{2}$ and $s_{2}$ we used SchOONSCHIP. The final result can be expressed in logarithms and Euler $n$ logarithms for $n=2$ and 3 . These functions can be defined as follows:

$$
\begin{aligned}
& \operatorname{Li}_{2}(z)=-\int_{0}^{z} \frac{\ln (1-x)}{x} d x \\
& \operatorname{Li}_{3}(z)=\int_{0}^{z} \frac{\operatorname{Li}_{2}(x)}{x} d x
\end{aligned}
$$

Our final result is

$$
\begin{aligned}
\frac{d \sigma^{\alpha q}}{d Q^{2}}= & \frac{4 \pi \alpha^{2}}{3 s Q^{2}}\left(\frac{\alpha_{s}}{\pi}\right)^{2} \frac{N^{2}-1}{16 N^{2}} e_{2}{ }^{2} \\
\times & {\left[(1+\tau) \ln \tau+\frac{1}{2}-\frac{1}{2} \tau-\frac{2}{3} \tau^{2}+\frac{2}{3} \tau^{-1}\right] \ln ^{2}\left(\frac{-p_{1}{ }^{2}}{Q^{2}} \frac{\tau^{2}}{1-\tau}\right) } \\
& +\left[-(1+\tau) \ln ^{2} \tau+\left(\tau-\frac{4}{3} \tau^{-1}\right) \ln \tau-2(1+\tau) \operatorname{Li}_{2}(1-\tau)+\frac{14}{3}-\frac{11}{3} \tau-\frac{4}{3} \tau^{2}+\frac{1}{3} \tau^{-1}\right] \ln \left(\frac{-p_{1}^{2}}{Q^{2}} \frac{\tau^{2}}{1-\tau}\right) \\
& +(1+\tau)\left[6 \operatorname{Li}_{3}(1-1 / \tau)+4 \operatorname{Li}_{3}(1-\tau)-2 \ln (\tau) \operatorname{Li}_{2}(1-\tau)-\frac{1}{3} \ln ^{3} \tau\right]+\left(3-4 \tau+\frac{4}{3} \tau^{-1}\right) \operatorname{Li}_{2}(1-\tau) \\
& \left.+\left(\frac{3}{2}-2 \tau+\frac{2}{3} \tau^{-1}\right) \ln ^{2} \tau+\left(\frac{15}{2}+5 \tau-\frac{1}{3} \tau^{-1}\right) \ln \tau+\frac{79}{4}-\frac{115}{6} \tau-\frac{13}{12} \tau^{2}+\frac{1}{2} \tau^{-1}\right] .
\end{aligned}
$$

All parameters are defined in the usual way:

$$
s=\left(p_{1}+p_{2}\right)^{2} \quad \tau=Q^{2} / s \quad \alpha=e^{2} / 4 \pi \quad \alpha_{s}=g_{s}^{2} / 4 \pi .
$$

The factor $\left(N^{2}-1\right) / 16 N^{2}$ contains the color factor for an SU(N) gauge theory and a spin-average factor $\frac{1}{4}$. The leading logarithms in (3.7) agree with results given elsewhere. ${ }^{9,10}$ Of course, a contribution with $e_{2}^{2}$ replaced by $e_{1}^{2}$, and $p_{1}{ }^{2}$ by $p_{2}^{2}$ should be added to (3.7).

\section{B. Interference terms}

For the interference terms the relatively simple calculation described above does not work. We have evaluated the integral in a straightforward but tedious way by integrating over all angles first, and finally integrating over the energies of the outgoing quarks.

When single diagrams are calculated this way one finds double and single mass singularities, which cancel in the sum of all diagrams, as expected.

This integration procedure has the advantage of being rather independent of the topology of the diagrams considered, as long as the phase space for the final states is the same. For instance, it can be used to calculate the squared terms as well, although this is much more complicated than the procedure described in Sec. III A. This provided a valuable and almost completely independent check of (3.7).

For the interference terms we find the following result:

$$
\begin{aligned}
\frac{d \sigma^{I}}{d Q^{2}}= & \frac{4 \pi \alpha^{2}}{3 s Q^{2}}\left(\frac{\alpha_{s}}{\pi}\right)^{2} \frac{N^{2}-1}{16 N^{2}} e_{1} e_{2} \\
& \times\left\{\tau^{-1}\left[1+(1+\tau)^{2}\right]\left[4 \ln ^{3}(1+\tau)-2 \pi^{2} \ln (1+\tau)-10 \ln ^{2} \tau \ln (1+\tau)-8 \mathrm{Li}_{3}\left(1-\frac{1}{\tau}\right)-24 \mathrm{Li}_{3}\left(\frac{1}{1+\tau}\right)\right]\right. \\
& +(1+\tau)\left[-20 \mathrm{Li}_{2}(-\tau)-\frac{5}{3} \pi^{2}-20 \ln \tau \ln (1+\tau)\right]+\frac{8}{3}\left(1+\tau+\tau^{-1}\right) \ln \ln ^{3}+\left[20+16 \tau-\frac{1}{3} \pi^{2} \tau-\frac{10}{3} \pi^{2}\right] \ln \tau \\
& +\left(-16 \tau-40 \tau^{-1}\right) \ln \tau \mathrm{Li}_{2}(-\tau)+(8 \tau-10) \mathrm{Li}_{2}(1-\tau)+\left(-40+12 \tau+40 \tau^{-1}\right) \mathrm{Li}_{3}(-\tau)+13 \tau \ln ^{2} \tau \\
& \left.+\left(-4-14 \tau-24 \tau^{-1}\right) \mathrm{Li}_{3}(1-\tau)+\left(12+30 \tau+72 \tau^{-1}\right) \mathrm{Li}_{3}(1)+\left(-4+10 \tau+16 \tau^{-1}\right) \ln \tau \mathrm{Li}_{2}(1-\tau)+40(1-\tau)\right\} .
\end{aligned}
$$




\section{On-shell calculations}

For calculations with on-shell in-going quarks the same methods can be used. Owing to the fact that the result is now gauge-invariant, the analysis analogous to (3.1) $-(3.6)$ is slightly simplified and mass ratios as described in Sec. III A do not appear in these steps of the calculation. However, they do appear for other reasons. The kinematics is much more complicated for particles with nonzero mass, and causes linear combinations of the masses to appear in the arguments of the logarithms. For arbitrary values of the mass ratio an analytic final expression cannot be obtained. This situation is very much improved if one of the masses is set equal to zero. In the process we consider, the limit $m_{1} / m_{2} \rightarrow 0$ turns out to exist, and a result comparable to (3.7) can be obtained. The limit $m_{1} / m_{2} \rightarrow 0$ of course does not exist, because $m_{1}{ }^{2}$ is as essential for the mass singularities as $-p_{1}{ }^{2}$ in the off-shell case. We will not give the complete result of the on-shell calculation, but one aspect is worth mentioning. As one can see in (3.7), all the logarithms of $(1-\tau)$, important for the behavior of the constant terms near $\tau=1$, can be factored out together with the mass singularities. ${ }^{10}$ The same observation can be made for the on-shell result, with $m_{2} / m_{1} \rightarrow 0$. In that case, one finds factors

$$
\ln \left(\frac{m_{1}^{2}}{Q^{2}} \frac{\tau}{(1-\tau)^{2}}\right)
$$

instead of

$$
\ln \left(\frac{-p_{1}^{2}}{Q^{2}} \frac{\tau^{2}}{1-\tau}\right)
$$

Notice that, because the leading mass singularities are identical, the factor in front of $\ln ^{2}(1-\tau)$ is four times larger in the on-shell than in the off-shell case. This shows once more that phenomenological consequences cannot be drawn from these diagrams alone.

The appearance of $\ln (1-\tau)$ terms is precisely the same in the quark-gluon contribution. The result, calculated with an off-shell gluon and a massive quark, is given by (5.13).

\section{CALCULATION OF THE DEEP-INELASTIC SCATTERING DIAGRAMS}

The contribution of the diagrams of Fig. 1 to the structure function $W_{2}$ can be calculated with the method described in Sec. III A. In this case we consider first the subprocess $g\left(p^{\mu}\right)+\gamma\left(q^{\mu}\right)$ $\rightarrow q\left(p_{4}^{\mu}\right)+\bar{q}\left(p_{2}^{\mu}\right)$. The four terms contributing to this process add up to a tensor $B_{\mu \nu \rho \sigma}$, where $\mu$, $\nu$ are indices belonging to the photon, and $\rho, \sigma$ are gluon indices. The contribution to the parton structure function may then be written as follows:

$$
\begin{aligned}
\nu W_{2}^{j}\left(x_{p}, Q^{2}\right)=C \int d \odot[ & -\frac{1}{2} x_{p}\left(g^{\mu \nu}-\frac{12 x_{p}^{2}}{Q^{2}} p_{1}^{\mu} p_{1}^{\nu}\right) A^{\nu \sigma}\left(p_{1}, p_{3}\right) \\
& \left.\times B_{\mu \nu \rho \sigma}\left(p, q, p_{2}, p_{4}\right)\right] .
\end{aligned}
$$

Here $\nu W_{2}^{j}$ is the structure function of a parton of type $j, C$ contains all trivial factors, and $d \odot$ denotes the phase-space integral. The first tensor in (4.1) projects out $\nu W_{2}$, and the second one is defined by (3.2). The parton scaling variable $x_{p}$ is defined as $x_{p}=Q^{2} / 2 p \cdot q$.

From (4.1) the hadron structure functions can be obtained by means of the convolution

$$
\nu W_{2}\left(x, Q^{2}\right)=\sum_{j} \int_{x}^{1} d \xi f_{j}(\xi) W_{2}^{j}\left(x / \xi, Q^{2}\right) .
$$

As in Sec. III A, we now perform the phase-space integral for the subprocess. This integral yields a tensor $T_{\mu \nu \rho_{\sigma}}(p, q)$ which has to satisfy, because of gauge invariance,

$$
\begin{aligned}
& q^{\mu} T_{\mu \nu \rho \sigma}=q^{\nu} T_{\mu \nu \rho_{\sigma}}=0, \\
& p^{\rho} T_{\mu \nu \rho_{\sigma}}=p^{\sigma} T_{\mu \nu \rho_{\sigma}}=0 .
\end{aligned}
$$

Moreover, we are only interested in that part of the tensor which has the following symmetry properties:

$$
\begin{aligned}
& T_{\mu \nu \rho \sigma}=T_{\nu \mu \rho \sigma}, \\
& T_{\mu \nu \rho \sigma}=T_{\mu \nu \sigma \rho} .
\end{aligned}
$$

It can be shown that because of (4.3) and (4.4), the symmetric part of $T_{\mu \nu \rho \sigma}$ must be a linear combination of the following six tensors:

$$
\begin{aligned}
& T_{\mu \nu \rho \sigma}^{1}=G_{\mu \nu} H_{\rho \sigma}, \\
& T_{\mu \nu \rho \sigma}^{2}=G_{\mu \nu} P_{\sigma} P_{\sigma}, \\
& T_{\mu \nu \rho \sigma}^{3}=Q_{\mu} Q_{\nu} H_{\rho \sigma}, \\
& T_{\mu \nu \rho \sigma}^{4}=Q_{; L} Q_{\nu} P_{\rho} P_{\sigma}, \\
& T_{\mu \nu \rho \sigma}^{5}=F_{\mu \sigma} F_{\nu \sigma}+F_{\nu \sigma} F_{\mu \sigma}, \\
& T_{\mu \nu \rho \sigma}^{6}=F_{\mu \rho} Q_{\nu} P_{\sigma}+F_{\nu \rho} Q_{\mu} P_{\sigma}+F_{\mu \sigma} Q_{\nu} P_{\rho}+F_{\nu \sigma} Q_{\mu} P_{\rho},
\end{aligned}
$$

where

$$
\begin{aligned}
& G_{\mu \nu}=g_{\mu \nu}-\frac{q_{\mu} q_{\nu}}{q^{2}}, H_{\mu \nu}=g_{\mu \nu}-\frac{p^{\mu} p^{\nu}}{p^{2}}, \\
& P_{\mu}=q_{\mu}-\frac{p \cdot q}{p^{2}} p_{\mu}, \quad Q_{\mu}=p_{\mu}-\frac{p \cdot q}{q^{2}} q_{\mu}, \\
& F_{\mu \nu}=g_{\mu \nu}-\frac{p_{\mu} q_{\nu}}{p \cdot q} .
\end{aligned}
$$

Now one has to construct six operators to project out the structure functions belonging to these ten- 
sors. These operators can then be contracted with $B_{\mu \nu \rho \sigma}$, and the phase-space integral for the subprocess can be calculated to obtain the structure functions. They are combined with the tensors (4.5) and inserted in (4.1). The remaining two-di- mensional integral [comparable to (3.6)] is easier than the one calculated in Sec. III A, but still requires an algebraic program to handle the large number of terms. We finally obtain the following result:

$$
\begin{aligned}
\nu W_{2}^{\alpha}\left(x, Q^{2}\right)=\frac{N^{2}-1}{16 N}\left(\frac{\alpha_{s}}{\pi}\right)^{2} e_{2}{ }^{2} 2 x\{ & {\left[(1+x) \ln x+\frac{1}{2}-\frac{1}{2} x-\frac{2}{3} x^{2}+\frac{2}{3} x^{-1}\right] \ln ^{2}\left(\frac{-p_{1}{ }^{2}}{Q^{2}}\right) } \\
+ & {\left[3(1+x) \ln ^{2} x+\left(5+5 x-\frac{8}{3} x^{2}+\frac{4}{3} x^{-1}\right) \ln x+9-6 x-\frac{26}{9} x^{2}-\frac{1}{9} x^{-1}\right] \ln \left(\frac{-p_{1}{ }^{2}}{Q^{2}}\right) } \\
+ & 2(1+x) \ln ^{3}(x)+\left(8+13 x-\frac{4}{3} x^{2}+\frac{4}{3} x^{-1}\right) \ln ^{2} x+\left(\frac{58}{3}+\frac{4}{3} x-\frac{52}{9} x^{2}-\frac{1}{9} x^{-1}\right) \ln x \\
+ & \left.\frac{38}{3}-\frac{23}{3} x-\frac{163}{27} x^{2}+\frac{28}{27} x^{-1}+\left(\frac{4}{3} x^{2}+4\right) \frac{\pi^{2}}{6}+\frac{4}{3}(1+x)^{3} x^{-1}\left[\mathrm{Li}_{2}(-1 / x)-\ln (1+x) \ln x\right]\right\} .
\end{aligned}
$$

This is the result of an off-shell calculation. The on-shell calculation can be done in essentially the same way, at least at the limit $m_{2} / m_{1} \rightarrow 0$ (where $m_{2}$ is the mass of the quark to which the photon couples). We will not give the result, but the most important difference is the appearance of factors $\ln (1-x)$, which can be absorbed in the mass singularities if $\left.\ln \left[m_{1}{ }^{2} / Q^{2}\right)(1-x)\right]$ is used instead of $\ln \left(m_{1}{ }^{2} / Q^{2}\right)$. Also in this case, the appearance of factors $\ln (1-x)$ terms turns out to be related to the mass singularities and the regularization method, as was mentioned in Sec. IIIC for the $\ln (1-\tau)$ terms.

To get a completely independent check of the result, we have also calculated it with the method described in Sec. III B. As expected, this turned out to be much more complicated, but the final expression (4.6) was found to be correct.

\section{THE QUARK-GLUON TRANSITION}

For the subtraction of the nonleading mass singularities in (3.7) one only needs the leading logarithm of the quark-gluon transition, which is well known. Nonsingular terms in this transition function will influence the final result of the $q q$ contribution but, as we discussed in Sec. II, they can only be calculated meaningfully if a clear definition of the gluon distribution is given. However, to investigate the dependence of our final result upon the regularization method (i.e. massive, on-shell or massless, off-shell initial quarks), we need to know the relation between the constants if different regularization methods are used.

To achieve this we use essentially a slight extension of the method used in Ref. 11 to calculate the leading logarithms of the transition functions. We consider a process consisting of the radiation of a gluon by a quark, followed by a process of the type $g+B \rightarrow$ anything. Since the relation between on-shell and off-shell $q g$ transition functions is expected to be independent of the subprocess one considers, just as is the leading mass singularity, the simplest choice is the best one here. We choose both $B$ and "anything" to be a massless scalar particle, coupling to the gluon via a current $\left\langle p_{4}\left|J^{\nu, b}\right| q\right\rangle=\left\langle p_{4}\left|J^{\nu}\right| q\right\rangle T^{b}$. Here $\nu$ and $b$ are the Lorentz and color index of the gluon, and $T^{b}$ is a generator of the gauge group. The momenta are defined in Fig. 2. When the trivial $p_{4}$ integral has been performed, the squared matrix element can be written as follows:

$$
g^{2} A^{\mu \nu}\left(p_{1}, p_{3}\right) T_{\mu \nu}(p, q) \operatorname{Tr}\left(T^{a} T^{b}\right) \operatorname{Tr}\left(T^{a} T^{b}\right) .
$$

The conserved tensor $T_{\mu \nu}$ consists of two terms:

$$
\begin{aligned}
T_{\mu \nu}=\delta\left((p+q)^{2}\right)[ & T_{1}\left(-g_{\mu \nu}+\frac{p_{\mu} p_{\nu}}{p^{2}}\right) \\
& \left.+T_{2}\left(q_{\mu}-\frac{p \cdot q}{p^{2}} p_{\mu}\right)\left(q_{\nu}-\frac{p \cdot q}{p^{2}} p_{\nu}\right)\right] .
\end{aligned}
$$

The appearance of two terms reflects the fact that the off-shell gluon has three polarizations, two of which are transversal and one longitudinal. The longitudinal polarization cannot be treated on the same basis as the other two, and we therefore explicitly distinguish between them by introducing two kinds of distribution functions $f^{T}$ and $f^{L}$. In the c.m. frame of the subprocess $g B \rightarrow B$, with $\vec{p}$ and $\overrightarrow{\mathrm{q}}$ directed along the $z$ axis, the polarization vectors can be chosen as follows:

$$
\begin{aligned}
& \epsilon_{\mu}^{T, 1}=(0,1,0,0), \\
& \epsilon_{\mu}^{T, 2}=(0,0,1,0), \\
& \epsilon_{\mu}^{L}=\frac{1}{\left[(p \cdot q)^{2} / p^{2}-q^{2}\right]^{1 / 2}}\left(q_{\mu}-\frac{p \cdot q}{p^{2}} p_{\mu}\right) .
\end{aligned}
$$

We re-express (5.2) in terms of two tensors orthogonal to $\epsilon_{\mu}^{T}$ and $\epsilon_{\mu}^{L}$, respectively: 


$$
\begin{aligned}
T_{\mu \nu}(p, q)=\delta\left((p+q)^{2}\right)\{ & T^{T}\left[\left(\frac{q^{2} p^{2}-(q \cdot p)^{2}}{q^{2}}\right)\left(-g^{\mu \nu}+\frac{p^{\mu} p^{\nu}}{p^{2}}\right)+\frac{p^{2}}{q^{2}}\left(q_{\mu}-\frac{p \cdot q}{p^{2}} p_{\mu}\right)\left(q_{\nu}-\frac{p \cdot q}{p^{2}} p_{\nu}\right)\right] \\
+ & T^{L}\left[-\frac{p^{2}}{q^{2}}\left(q_{\mu}-\frac{p \cdot q}{p^{2}} p_{\mu}\right)\left(q_{\nu}-\frac{p \cdot q}{p^{2}} p_{\nu}\right)\right\} .
\end{aligned}
$$

Now we can derive the $g B$ cross sections for transversal and longitudinal gluons,

$$
\begin{aligned}
\sigma^{T} & =\frac{1}{2} \frac{1}{N^{2}-1} \frac{1}{4 p \cdot q} \sum_{\lambda=1}^{2} \epsilon_{\mu}^{T, \lambda} \epsilon_{\nu}^{* T, \lambda} T^{\mu \nu}(p, q) \operatorname{Tr}\left(T^{a} T^{a}\right) \\
& =\frac{1}{16 Q^{2}} \delta\left(1-x_{p}\right) T^{T} \\
\sigma^{L} & =\frac{1}{N^{2}-1} \frac{1}{4 p \cdot q} \epsilon_{\mu}^{L} \epsilon_{\nu}^{* L} T^{\mu \nu}(p, q) \operatorname{Tr}\left(T^{a} T^{a}\right) \\
& =\frac{1}{16 Q^{2}} \delta\left(1-x_{p}\right) T^{L}
\end{aligned}
$$

(Of course, terms of order $p^{2} / Q^{2}$ are neglected here.) The hadronic cross section is obtained by means of the following convolution:

$\sigma_{H}(x)=\int_{x}^{1} d \xi\left[f^{L}(\xi) \sigma^{L}\left(\frac{x}{\xi}\right)+f^{T}(\xi) \sigma^{T}\left(\frac{x}{\xi}\right)\right]$.

Using (5.5)-(5.7) we obtain

$$
\sigma_{H}(x)=\frac{1}{16 Q^{2}}\left[x f^{L}(x) T^{L}+x f^{T}(x) T^{T}\right]
$$

This relation will be used to identify the longitudinal and transversal quark-gluon transition functions. We express the cross section for the process $q B \rightarrow q B$ in $T^{T}$ and $T^{L}$,

$$
\sigma^{\alpha}=\frac{1}{2} \frac{1}{N} \frac{1}{4 p_{1} \cdot q} 4 \pi \alpha_{s} \int \frac{d^{3} p_{3}}{(2 \pi)^{3} 2 E_{3}} A^{\mu \nu}\left(p_{1}, p_{3}\right) T_{\mu \nu}(p, q) \operatorname{Tr}\left(T^{a} T^{b}\right) \operatorname{Tr}\left(T^{a} T^{b}\right) .
$$

The result is

$$
\begin{aligned}
\sigma^{\alpha}=\frac{x_{p}}{16 Q^{2}} \frac{N^{2}-1}{4 N} \frac{\alpha_{s}}{\pi}\left\{T^{T}[\right. & \left.-4-\frac{3}{2 x_{p}}+\frac{\left(1-x_{p}\right)\left(\mu^{2}\left(1-2 / x_{p}\right)-2 m_{1}^{2}\right)}{m_{1}^{2} x_{p}+\mu^{2}\left(1-x_{p}\right)}-\left(\frac{\left(1-x_{p}\right)^{2}+1}{x_{p}}\right) \ln \left(\frac{m_{1}{ }^{2} x_{p}{ }^{3}}{Q^{2}\left(1-x_{p}\right)}+\frac{\mu^{2} x_{p}{ }^{2}}{Q^{2}}\right)\right] \\
+ & T^{L}\left[2+\frac{2}{x_{p}}-\frac{1}{4} \mu^{4} \frac{\left(1 / x_{p}\right)\left(1-x_{p}\right)^{2}}{\left[m_{1}^{2} x_{p}+\mu^{2}\left(1-x_{p}\right)\right]^{2}}+\mu^{2} \frac{\left(2 / x_{p}-\frac{3}{2}\right)\left(1-x_{p}\right)}{m_{1}^{2} x_{p}+\mu^{2}\left(1-x_{p}\right)}\right. \\
& \left.\left.+\left(\frac{2}{x_{p}}-2\right) \ln \left(\frac{m_{p}^{2} x_{p}}{Q^{2}\left(1-x_{p}\right)}+\frac{\mu^{2} x_{p}^{2}}{Q^{2}}\right)\right]\right\} .
\end{aligned}
$$

Here $\mu^{2}=m_{1}{ }^{2}-p_{1}{ }^{2}$. This result must now be convoluted with the quark distribution function and added to (5.8). The resulting expression is then reinterpreted as an expression similar to (5.8), but with $f^{L}$ and $f^{T}$ replaced by $Q^{2}$-dependent distribution functions. The factors, appearing in front of $T^{T}$ and $T^{L}$, are the distribution functions for transversal and longitudinal gluons. Although this procedure yields a specific value for the nonsingular terms in the quark-gluon transition function, we are not obliged to choose this value which is of course completely dependent on the subprocess we consider. Therefore, we keep only the logarithmic term in the off-shell case. For the transveral gluons we then find

$$
f_{g}^{T}\left(x, Q^{2}\right)=f_{g}^{T}(x)+\frac{N^{2}-1}{4 N} \frac{\alpha_{s}}{\pi} \int_{x}^{1} \frac{f_{\alpha}(\xi)}{\xi}\left[-\left(\frac{(1-\eta)^{2}+1}{\eta}\right) \ln \left(\frac{-p_{1}^{2} \eta^{2}}{Q^{2}}\right) d \xi\right],
$$

where $\eta=x / \xi$.

As we discussed in Sec. II, it is not yet clear which, if any, of the gluon distributions given in the literature are in agreement with out definition (5.11), but our final result can easily be modified if a different definition is favored. To get a rough impression of the effect of different choices, one can calculate the gluon distribution from a perturbative QCD relation like (5.11), assuming that it vanishes at a certain point $p_{1}{ }^{2}=-Q_{0}{ }^{2}$. If, for instance, all the constants in (5.10) are taken into account, this procedure would yield a negative gluon-distribution function, except for very high values of $Q^{2}$. This conflicts with the usual interpretation of the distribution functions. The correct approach would be to use renormalization-group-improved transition functions, as was done for the dynamically calculated gluon distribution of Ref..$^{12}$, but then one has to go beyond the leading order to see the effect of different conventions. We will not do that in this paper, but this point may be worth further investigation.

Once the convention is fixed by (5.11), the corresponding on-shell result can be calculated unambiguously . Using (5.10) and (5.11) we find 


$$
f_{g}^{T}\left(x, Q^{2}\right)=f_{g}^{T^{\prime}}(x)+\frac{N^{2}-1}{4 N} \frac{\alpha}{\pi} \int_{x}^{1} \frac{f_{g}^{\prime}(\xi)}{\xi}\left[-\left(\frac{(1-\eta)^{2}+1}{\eta}\right) \ln \left(\frac{m_{1}^{2} \eta^{3}}{Q^{2}(1-\eta)}\right)+1\right] .
$$

The primes indicate that the "unrenormalized" distribution functions depend upon the set of conventions used to regularize the mass singularities. The left-hand sides of Eqs. (5.11) and (5.12), however, are identical to a given order in perturbation theory.

The difference between (5.11) and (5.12) is the basic result of this section. The same result can also be derived by calculating the quark-quark transition function in the axial gauge of the gluon. The quark-gluon transition function is obtained from the latter by replacing $x_{p}$ by $\left(1-x_{p}\right)$. Of course, only the $m_{1}{ }^{2}$ and $\mu^{2}$ dependence of (5.10) is reproduced in this case, not the constant terms.

From (5.10) and (5.8) one can in exactly the same way derive the transition function for a longitudinal polarized gluon, but we will not need it. To show that they are unnecessary, the quark-gluon contribution to the Drell-Yan process must be calculated with an off-shell gluon for transversal and longitudinal gluons separately. The results are

$$
\begin{aligned}
& \frac{d \sigma^{L}}{d Q^{2}}=\frac{4 \pi \alpha^{2}}{3 s Q^{2}} \frac{\alpha_{s}}{\pi} \frac{1}{8 N} e_{q}{ }^{2}\left[\frac{8 p^{2} \tau^{2}(1-\tau)^{2}}{m^{2}-p^{2} \tau(1-\tau)}\right], \\
& \frac{d \sigma^{T}}{d Q^{2}}=\frac{4 \pi \alpha^{2}}{3 s Q^{2}} \frac{\alpha_{s}}{\pi} \frac{1}{8 N} e_{q}{ }^{2}\left[-2\left[(1-\tau)^{2}+\tau^{2}\right] \ln \left(\frac{m_{1}{ }^{2} \tau}{Q^{2}(1-\tau)^{2}}-\frac{p^{2}}{Q^{2}} \frac{\tau^{2}}{1-\tau}\right)+(1-\tau)\left(1+3 \tau+\frac{2 p^{2} \tau-4 p^{2} \tau^{2}(1-\tau)+4 m^{2} \tau}{m^{2}-p^{2} \tau(1-\tau)}\right)\right] .
\end{aligned}
$$

Here $p^{\mu}$ is the momentum of the gluon. To get the physical contribution of the quark-gluon process, the singularities must be subtracted. To this end, the gluon contribution to $\nu W_{2}$ has to be calculated and absorbed in the (anti)quark distribution according to relation (2.7). Then one finds the following result:

$$
\begin{aligned}
f_{q}\left(x, Q^{2}\right)=f_{q}(x)+\frac{\alpha_{s}}{4 \pi} \int_{x}^{1} d \xi \frac{f_{g}^{T}(\xi)}{\xi}\{ & \left.-\left[(1-\eta)^{2}+\eta^{2}\right] \ln \left[\left(\frac{m^{2} \eta}{1-\eta}-p^{2} \eta^{2}\right) / Q^{2}\right]\right] \\
& \left.+6 \eta-6 \eta^{2}-1-\eta(1-\eta)\left[\frac{p^{2}-2 p^{2} \eta(1-\eta)+2 m^{2}}{p^{2} \eta(1-\eta)-m^{2}}\right]\right\} \\
+ & \frac{\alpha_{s}}{4 \pi} \int_{x}^{1} d \xi \frac{f_{g}^{L}(\xi)}{\xi}\left[\frac{-4 p^{2} \eta^{2}(1-\eta)^{2}}{p^{2} \eta(1-\eta)-m^{2}}\right]
\end{aligned}
$$

When the distributions of the leading $q \bar{q}$ process are made scale-dependent according to (5.14), the terms proportional to $f^{L}$ vanish in the remaining finite terms. This implies that the effective quark quark-gluon cross section for longitudinal gluons is zero. The terms proportional to $f^{T}$ yield the $q g$ cross section calculated in Ref. 5:

$$
\begin{gathered}
\frac{d \sigma^{\alpha g}}{d Q^{2}}=\frac{4 \pi \alpha^{2}}{3 Q^{4}} \frac{\alpha_{s}}{\pi} \frac{e_{q}^{2}}{4 N} \tau \\
\tau\left\{\left[(1-\tau)^{2}+\tau^{2}\right] \ln (1-\tau)\right. \\
\left.+\frac{3}{2}-5 \tau+\frac{9}{2} \tau^{2}\right\} .
\end{gathered}
$$

This proves, for the $q g$ process, that the final result is independent of the way the mass singularities are regularized.

\section{SUBTRACTION OF THE SINGULAR TERMS}

To obtain the final result we must now combine the expressions obtained in Secs. III-V. The result of Sec. IV is

$$
\nu W_{2}^{q}\left(x, Q^{2}\right)=\frac{N^{2}-1}{16 N}\left(\frac{\alpha_{s}}{\pi}\right)^{2} e_{2}{ }^{2} W\left(x, p_{1}{ }^{2} / Q^{2}\right) .
$$

The function $W$ is defined in (4.6). The contribution of (6.1) to the hadron structure can be calculated with (4.2). To absorb this contribution in the (anti)quark contribution function we use, besides (2.7), the natural convention that the amount of quarks and antiquarks in the sea must be equal. Then one immediately obtains

$$
\begin{aligned}
\bar{f}_{i}\left(x, Q^{2}\right)= & \bar{f}_{i}(x)+\frac{\alpha_{s}}{\pi} \int_{x}^{1} d \xi \frac{f_{g}(\xi)}{\xi} T_{g \bar{q}_{i}}\left(\eta, \frac{p^{2}}{Q^{2}}\right) \\
& +\left(\frac{\alpha_{s}}{\pi}\right)^{2} \int_{x}^{1} d \xi \frac{f_{j}(\xi)}{\xi} T_{\alpha_{j \bar{a}} i}\left(\eta, \frac{p^{2}}{Q^{2}}\right) .
\end{aligned}
$$

As before, $\eta=x / \xi$. The gluon-antiquark transition function $T_{g \bar{\alpha}_{i}}$ is defined in (5.14), but it is irrelevant for our purpose. The quark-antiquark transition function is related to $W$, i.e.,

$$
T_{a_{j} \overline{\bar{a}_{i}}}\left(x, \frac{p^{2}}{Q^{2}}\right)=\frac{N^{2}-1}{16 N} \frac{1}{2 \eta} W\left(\eta, \frac{p^{2}}{Q^{2}}\right) .
$$

The other subtraction which has to be made is due to the quark-gluon transition. According to (5.11), 


$$
\begin{aligned}
& f_{g}\left(x, Q^{2}\right)=f_{g}(x)+\frac{\alpha_{s}}{\pi} \int_{x}^{1} d \xi \frac{f_{g}(\xi)}{\xi} T_{q g}\left(\eta, \frac{p^{2}}{Q^{2}}\right) \\
& T_{q g}\left(\eta, \frac{p^{2}}{Q^{2}}\right)=-\frac{N^{2}-1}{4 N}\left[\frac{(1-\eta)^{2}+1}{\eta}\right] \ln \frac{-p^{2} \eta^{2}}{Q^{2}}
\end{aligned}
$$

The transition functions, defined in (6.2)-(6.5), are related by a Mellin transformation to the $\Gamma$ factors used in the formal arguments of Sec. II, but they differ by a finite renormalization. Therefore, the moments of these transition functions do not satisfy the normalization of the $\Gamma$ factors which can be derived from (2.3):

$$
\Gamma_{i j}^{n}(0)=\delta_{i j}
$$

It is however possible, but unnecessary, to redefine both the bare parton distribution functions and the transition functions in such a way that the $Q^{2}-$ dependent distribution functions are unchanged, and the moments of the transition functions are equal to the ones defined by (2.3) at the renormalization point. Then $(6.6)$ is automatically satisfied.

The transition functions must now be combined with the cross sections, which we denote as follows:

$$
\begin{aligned}
& \frac{d \sigma^{Q \bar{q}}}{d Q^{2}}=\frac{4 \pi \alpha^{2}}{3 Q^{4}} \frac{e_{q}^{2}}{N} \delta(1-\tau), \\
& \frac{d \sigma^{\alpha g}}{d Q^{2}}=\frac{4 \pi \alpha^{2}}{3 Q^{4}} \frac{e_{q}^{2}}{4 N} \frac{\alpha}{\pi} \tau \Sigma^{q g}(\tau), \\
& \frac{d \sigma^{\alpha_{1} q_{2}}}{d Q^{2}}=\frac{4 \pi \alpha^{2}}{3 Q^{4}}\left(\frac{\alpha_{s}}{\pi}\right)^{2} \frac{N^{2}-1}{16 N^{2}} \tau \\
& \times\left[e_{1}{ }^{2} \tilde{\Sigma}{ }^{\alpha Q}\left(\tau, \frac{p_{2}{ }^{2}}{Q^{2}}\right)+e_{2}{ }^{2} \tilde{\Sigma}^{\alpha Q}\left(\tau \frac{p_{1}{ }^{2}}{Q^{2}}\right)\right. \\
& \left.+2 e_{1} e_{2} \Sigma_{I}^{q Q}(\tau)\right] \text {. }
\end{aligned}
$$

The functions $\Sigma^{q g}, \tilde{\Sigma}^{q q}$, and $\Sigma_{I}^{q Q}$ are defined by (5.15), (3.7), and (3.8), respectively. The function $\tilde{\Sigma}^{q q}$ still contains mass singularities, whereas the other ones are finite. These singularities are now absorbed in the parton distribution functions according to (6.2), (6.4), and (2.1). In (6.9) $\tilde{\Sigma}^{a q}$ is then replaced by a nonsingular function $\Sigma^{\alpha \alpha}$ which equals

$$
\begin{aligned}
\frac{N^{2}-1}{16 N^{2}} \Sigma^{q a}(\tau)= & \frac{N^{2}-1}{16 N^{2}} \Sigma^{q Q}\left(\tau, \frac{p_{i}{ }^{2}}{Q^{2}}\right)-T_{q \bar{q}}\left(\tau, \frac{p_{i}{ }^{2}}{Q^{2}}\right) \\
& -\int_{\tau}^{1} \frac{d \xi}{\xi} T_{q g}\left(\xi, \frac{p_{i}{ }^{2}}{Q^{2}}\right) \frac{1}{4 N} \Sigma^{q g}(\tau / x) .
\end{aligned}
$$

We obtain the following result:

$$
\begin{aligned}
\Sigma^{q q}(\tau)= & \frac{401}{36}-\frac{241}{18} \tau+\frac{545}{108} \tau^{2}-\frac{151}{54} \tau^{-1}-\left(4+\frac{4}{3} \tau^{2}\right) \frac{\pi^{2}}{6}+\ln \tau\left(\frac{13}{2}-\frac{11}{3} \tau+\frac{28}{9} \tau^{2}-\frac{4}{9} \tau^{-1}\right) \\
& +\ln ^{2} \tau\left(-\frac{3}{2}-9 \tau-\frac{4}{3} \tau^{2}-\frac{2}{3} \tau^{-1}\right)-(1+\tau) \ln ^{3} \tau+\ln (1-\tau)\left(-\frac{14}{3}+\frac{11}{3} \tau-\frac{14}{9} \tau^{2}+\frac{23}{9} \tau^{-1}\right) \\
& +\ln (1-\tau) \ln \tau\left(2+3 \tau+\frac{8}{3} \tau^{2}+\frac{4}{3} \tau^{-1}\right)-(1+\tau) \ln (1-\tau) \ln ^{2} \tau+\ln ^{2}(1-\tau)\left(\frac{1}{2}-\frac{1}{2} \tau-\frac{2}{3} \tau^{2}+\frac{2}{3} \tau^{-1}\right) \\
& +(1+\tau) \ln ^{2}(1-\tau) \ln \tau+\left(4+4 \tau+\frac{4}{3} \tau^{2}+\frac{4}{3} \tau^{-1}\right)\left[\ln (1+\tau) \ln (\tau)-\mathrm{Li}_{2}(-1 / \tau)\right] \\
& +(1+\tau)\left[8 \operatorname{Li}_{3}(1-\tau)+10 \mathrm{Li}_{3}(1-1 / \tau)+2 \ln (1-\tau) \operatorname{Li}_{2}(1-\tau)-6 \ln \tau \operatorname{Li}_{2}(1-\tau)\right] \\
& +\operatorname{Li}_{2}(1-\tau)\left(7-2 \tau+4 \tau^{-1}\right) .
\end{aligned}
$$

Exactly the same result has been obtained with the on-shell results, which first of all show that the result is indeed unambiguous, but also provide an important check of the calculations.

The cancellation of the singular terms, not only in leading but also in nonleading order, shows that the correct variable for the description of scaling deviations for the Drell-Yan process is $Q^{2}$. This implies that the same distribution functions $f_{i}\left(x, Q^{2}\right)$ must be used in both the Drell-Yan process and in deep-inelastic scattering. However, whether $Q^{2}$ is the correct variable for the running coupling constant, determined in deep-inelastic scattering, remains an open question, which can only be answered by the calculation of two-loop insertions.

\section{NUMERICAL RESULTS AND CONCLUSIONS}

The expressions for $\Sigma^{\alpha \alpha}(\tau)$ and $\Sigma_{I}^{\alpha \alpha}(\tau)$, which are the basic results of this paper, are plotted in Fig. 4. The different behavior near $\tau=1$ of these functions is mainly due to the absence of $\ln (1-\tau)$ terms in the contribution of the interference terms. An expansion in $(1-\tau)$ yields

$$
\begin{aligned}
& \sum^{q q}(\tau) \sim(1-\tau)\left[\frac{1}{2} \ln ^{2}(1-\tau)-3 \ln (1-\tau],\right. \\
& \sum_{I}^{q \alpha}(\tau) \sim-\frac{7}{8}(1-\tau)^{2} .
\end{aligned}
$$

At this point we can compare our results with the ones reported in Ref. 10. Unfortunately, these authors have given a plot of their results and the behavior near $\tau=1$, but not a complete analytic expression, which makes a detailed comparison 


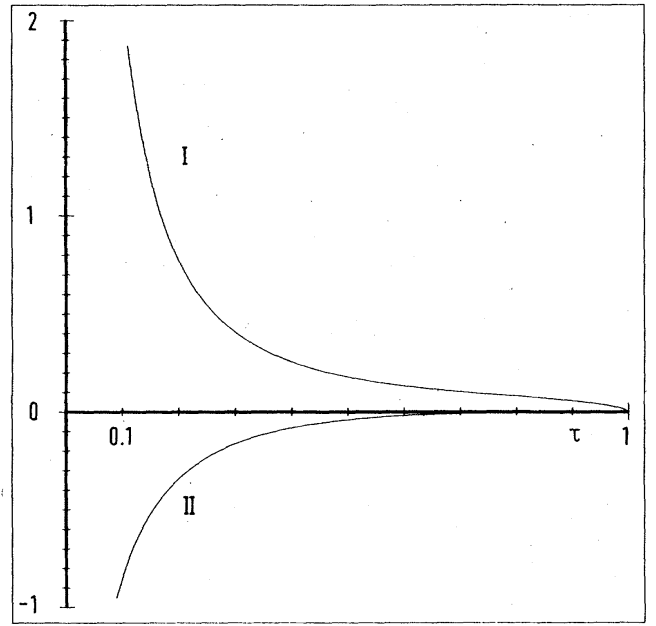

FIG. 4. Plot of the function $\left(N^{2}-1\right) /\left(16 N^{2}\right) \Sigma^{\alpha q}(\tau)$ (curve I), and $\left(N^{2}-1\right) /\left(16 N^{2}\right) \Sigma_{I}^{q q}(\tau)$ (curve II), with $N=3$.

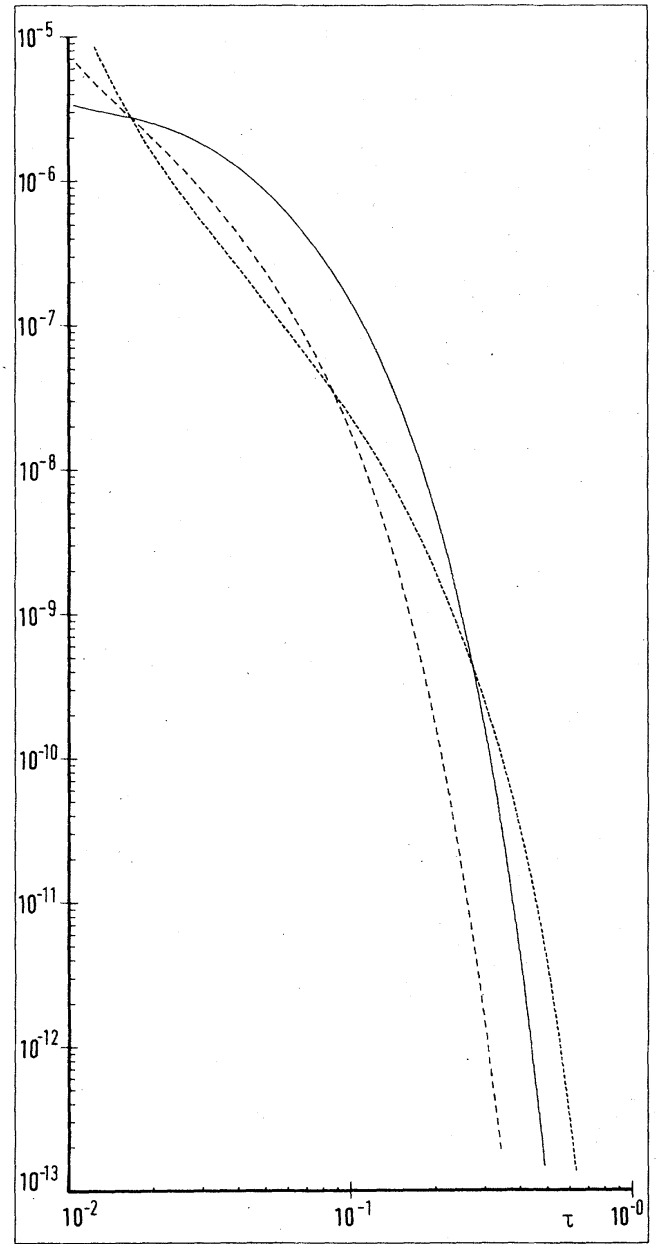

FIG. 5. Plot of $Q^{4}\left(d \sigma / d Q^{2}\right)$ (in $\left.\mathrm{mb} \mathrm{GeV}^{2}\right)$ vs $\tau$ for $\sqrt{s}=6$ GeV. Solid line: Drell-Yan contribution; long-dashed line: magnitude of the quark-gluon contribution; shortdashed line: quark-quark contribution. rather difficult. Moreover, they use a different subtraction of the mass singularities, both for the gluon and for the antiquark distribution function. For the interference term a result proportional to $(1-\tau)^{3}$ is reported in Ref. 10, but nevertheless the numerical agreement with our results is good.

To compare the results for the other term, we have applied the subtraction of Ref. 10 to our result (3.7). We were able to reproduce to limiting behavior near $\tau=1$ as given in Ref. 10, but we found a clear numerical discrepancy for small values of $\tau$. We share their observations concerning the combined appearance of mass singularities and factors $\ln (1-\tau)$ or $\ln (1-x)$, but we find a different factor in front of the nonleading $\ln (1-\tau)$ term, although we find the same mass singularities. ${ }^{13}$ This difference can be attributed to an extra constant which is added in Ref. 10 to the gluon transition function. This also illustrates that the aforementioned observation is mainly of mathematical interest.

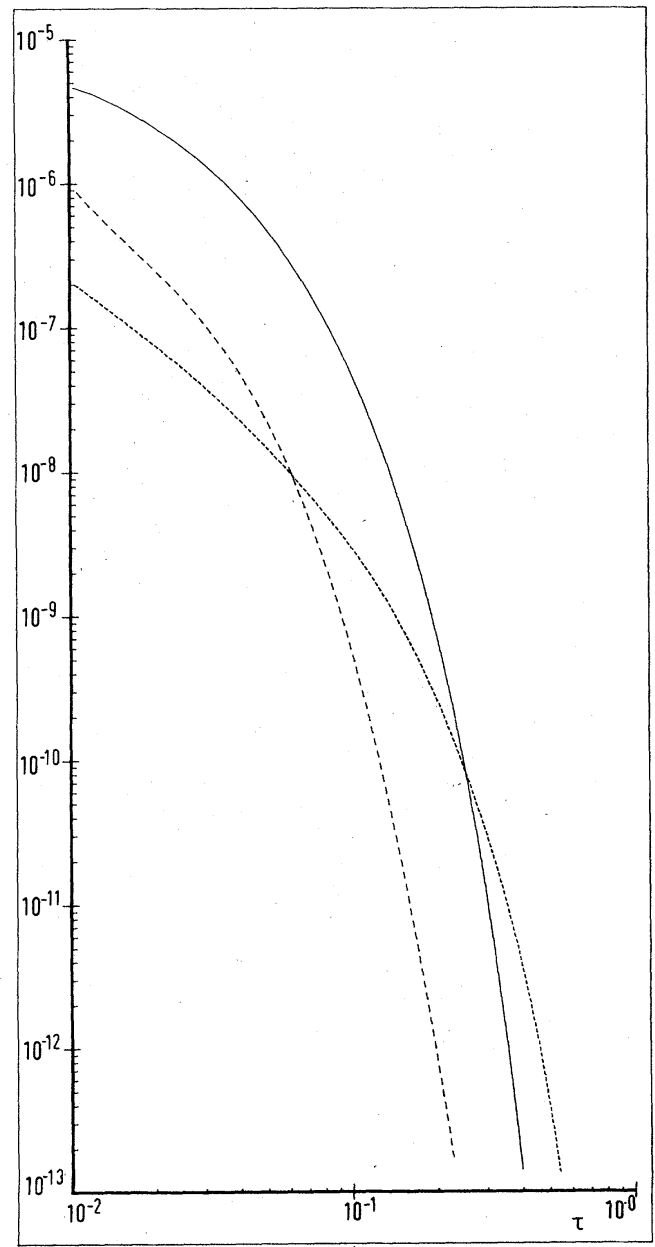

FIG. 6. As in Fig. 5 , but with $\sqrt{s}=27 \mathrm{GeV}$. 
To estimate the magnitude of the quark-quark contribution, we have convoluted our results with distribution functions determined in deep-inelastic scattering. Several parametrizations are available in the literature, but the valence-quark distributions are not very different. This means that the quark-quark contribution can be calculated accurately in the case of proton-proton collisions. The leading contribution and the quark-gluon contribution are much more difficult to calculate because of the differences in the parametrizations. Moreover, the sea and gluon distributions are very sensitive to the conventions used to subtract the singular terms. A correct calculation of the leptonpair production cross section in proton-proton scattering requires a much more accurate determination of the nonvalence distributions. Our numerical results must therefore be considered as order-of-magnitude estimates. For our calculations we have used the Buras-Gaemers distributions and $\alpha_{s}\left(Q^{2}\right)$ of Bardeen et al. ${ }^{7}$

We have calculated the leading $q \bar{q}$ contribution, the $q g$ contribution, and the $q q$ contribution. ${ }^{14}$ The results are plotted in Figs. 5 and 6 for $\sqrt{s}=6$ and $27 \mathrm{GeV}$, respectively. For small values of $\tau$, perturbation theory breaks down because the running coupling constant becomes too large. This explains the large $q g$ and $q q$ contribution near $\tau=1$ in Fig. 5. In all other cases the magnitude of the quark-gluon contribution is much smaller than the leading Drell-Yan process, but the quark-quark contribution completely overwhelms all other contributions for $\tau>0.4$. This is, of course, due to the fact that the nonvalence distributions vanish much faster than the valence distributions if $\tau$ approaches 1. If different distribution functions are used, this conclusion will remain qualitatively correct, but we expect the precise value of $\tau$, where the $q q$ process becomes larger than the other contributions, to be rather sensitive to the nonvalence distributions. The distributions of Ref. 12 and the scaling distributions of Ref. 15 lead to larger $q q$ and $q g$ contributions near $\tau=1$.

To observe the effect of quark-quark scattering in the total cross section for lepton-pair production, one obviously needs data at large values of $\tau$. Unfortunately, the cross section is very small in this region. Therefore, it will be very difficult to measure the expected deviation from the DrellYan formula.

\section{ACKNOWLEDGMENTS}

We are grateful to Dr. R. P. Van Royen for valuable remarks and critical comments, and to Dr. E. van Beveren and Dr. G. Austen for technical computer assistance. W. L. van Neerven thanks Dr. B. Humpert for useful discussions about this subject. Part of this work was included in the research program of the Stichting voor Fundamenteel Onderzoek der Materie (FOM), with financial support from the Nederlandse Organisatie voor Zuiver Wetenschappelijk Onderzoek (ZWO).
${ }^{1}$ H. D. Politzer, Nucl. Phys. B129, 301 (1977); D. Amati, R. Petronzio, and G. Veneziano, ibid. B140, 54 (1978); B146, 29 (1978); H. Georgi, M. Machacek, H. D. Politzer, and G. G. Ross, Phys. Lett. 78B, 281 (1978), and Nucl. Phys. B152, 285 (1979); S. Libby and G. Sterman, Phys. Lett. 78B, 618 (1978); Phys. Rev. D 18, 3252 (1978); C. T. Sachrajda, Phys. Lett. 73B, 185 (1978).

${ }^{2}$ S. D. Drell and T. M. Yan, Phys. Rev. Lett. 25, 316 (1970); Ann. Phys. (N.Y.) 66, 578 (1971).

${ }^{3}$ H. Georgi, Phys. Rev. D 17, 3010 (1978).

${ }^{4} \mathrm{~J}$. Kubar-André and F. E. Paige, Phys. Rev. D 19, 221 (1979); B. Humpert and W. L. van Neerven, Phys. Lett. 84B, 327 (1979); 85B, 293 (1979). G. Altarelli, R. K. Ellis, and G. Martinelli, Nucl. Phys. B157, 461 (1979); J. Abad and B. Humpert, Phys. Lett. 78B, 627 (1978); $80 \mathrm{~B}, 433$ (1979); K. Harada, T. Kaneko, and N. Sakai, Nucl. Phys. B155, 169 (1979).

${ }^{5} \mathrm{~J}$. Abad and B. Humpert, Phys. Lett. 80B, 286 (1979); J. Kubar-André and F. E. Paige, Phys. Rev. D 19, 221 (1978); G. Altarelli, R. K. Ellis, and G. Martinelli, Nucl. Phys. B143, 521 (1978); B146, 544 (1978); A. P. Contogouris and J. Kripfganz, Phys. Rev. D 19, 2207 (1979).

${ }^{6}$ We abbreviate $d \sigma^{i j} / d Q^{2}$ to $\sigma^{i j}$.
${ }^{7}$ E. G. Floratos, D. A. Ross, and C. T. Sachrajda, Nucl. Phys. B129, 66 (1978); B139, 545 (1978); Phys. Lett. 80B, 269 (1979); W. A. Bardeen, A. J. Buras, D. W. Duke, and T. Muta, Phys. Rev. D 18, 3998 (1978); A. N. Schellekens, Lett. Nuovo Cimento 24, 513 (1979).

${ }^{8}$ A. J. Buras and K. Gaemers, Nucl. Phys. B132, 249 (1978).

${ }^{9}$ C. T. Sachrajda, Phys. Lett. 73B, 185 (1978).

${ }^{10} \mathrm{~J}$. Kripfganz and A. P. Contogouris, Phys. Lett. 84B, 473 (1979); A. P. Contogouris and J. Kripfganz, Phys. Rev. D 20, 2295 (1979).

${ }^{11}$ G. Altarelli and G. Parisi, Nucl. Phys. B126, 298 (1977).

${ }^{12}$ J. F. Owens and E. Reya, Phys. Rev. D 17,3003 (1978).

${ }^{13}$ This is only the case for the second paper of Ref. 6 . In the first one, terms proportional to $p_{1}{ }^{2}$ have been neglected in the numerator. This affects the nonleading logarithms.

${ }^{14}$ We have omitted the important $q \bar{q} \rightarrow \gamma^{*} g$ contribution in these plots because it is not related by factorization to the $q q$ process.

${ }^{15}$ R. F. Peierls, T. L. Trueman, and L. L. Wang, Phys. Rev. D 16, 1397 (1977). 